\title{
Narrativa
}

\section{Michele COMETA, Cultura visuale. Una genealogia}

\section{Matteo Moca}

\section{(2) OpenEdition}

\section{Journals}

\section{Edizione digitale}

URL: https://journals.openedition.org/narrativa/469

DOI: $10.4000 /$ narrativa.469

ISSN: 2804-1224

\section{Editore}

Presses universitaires de Paris Nanterre

\section{Edizione cartacea}

Data di pubblicazione: 1 décembre 2021

Paginazione: 293-294

ISBN: 978-2-84016-485-2

ISSN: 1166-3243

\section{Notizia bibliografica digitale}

Matteo Moca, «Michele cometA, Cultura visuale. Una genealogia», Narrativa [Online], 43 | 2021, online dal 01 novembre 2021, consultato il 03 novembre 2022. URL: http://journals.openedition.org/narrativa/ 469 ; DOI: https://doi.org/10.4000/narrativa.469

Questo documento è stato generato automaticamente il 3 novembre 2022

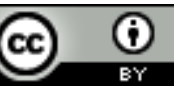

Creative Commons - Attribuzione 4.0 Internazionale - CC BY 4.0

https://creativecommons.org/licenses/by/4.0/ 


\title{
Michele cometa, Cultura visuale. Una genealogia
}

\author{
Matteo Moca
}

\section{NOTIZIA}

Michele comETA, Cultura visuale. Una genealogia, Milano, Raffaello Cortina, 2020, 345 p.

La cultura visuale, come annota Michele Cometa nell'introduzione al suo volume, è una disciplina che ha raggiunto una maturità teorica ed epistemologica. La sua natura di luogo di "una convergenza e di un dialogo attraverso i confini disciplinari", secondo la definizione di W.J.T. Mitchell, spalanca la complessità di una materia che Cometa chiama "indisciplina" proprio per il dialogo continuo tra codici diversi e convergenze tra campi disciplinari come la storia culturale, la storia dell'arte, la psicoanalisi, l'antropologia e la storia. Questo nuovo volume di Michele Cometa, tra gli studiosi che con maggiore continuità e profitto si sono occupati del tema (si vedano per esempio Archeologie del dispositivo. Regimi scopici della letteratura edito da Pellegrini nel 2016 o La scrittura delle immagini. Letteratura e cultura visuale pubblicato sempre da Raffaello Cortina nel 2011), mira, come suggerisce il sottotitolo, a fornire un quadro della disciplina in grado di enucleare tanto le origini della cultura visuale quanto gli sviluppi che si sono aperti e che la critica continua a percorrere, tenendo sempre presente come la cultura visuale sia "un oggetto di studio e nel contempo il metodo di studio da applicare a questo oggetto" e la complessità di una definizione in grado di comprendere le suggestioni teoriche afferenti a studiosi e geografie differenti (si può pensare per esempio alle relazioni tra visual studies britannici, la tedesca Bildwissenschaft, l'image science di Mitchell o la Bildanthropolgie di Hans Belting). Cometa organizza il suo percorso su una costellazione di autori, ordinando il suo ragionamento attorno ad Aby Warburg ("il vero innovatore della teoria dell'immagine"), Sigmund Freud ("estensore della più complessa teoria dello sguardo finora tentata") e Walter Benjamin ("grande archeologo del dispositivo"). L'analisi puntuale delle teorie e del lavoro di questi autori, nell'ottica di rintracciare la nascita dei ragionamenti e delle definizioni che innervano 
la disciplina, è anticipata da un primo capitolo introduttivo, "Vicende di un'indisciplina", utile per un inquadramento teorico e pratico (sono molte le immagini riprodotte), che ripercorre la nascita di questo campo di studi: si tratta di un capitolo denso che permette di cogliere anche il valore didattico del libro, arricchito da una serie di box che riportano alcuni brani classici della cultura visuale contemporanea. I due capitoli successivi, "Ricordi d'infanzia" e "Iconoteche", si occupano rispettivamente di mostrare come l'universo delle immagini attraversi l'esperienza biografica di Warburg, Freud e Benjamin e gli ambienti visuali in cui si sono mossi questi autori, affidando proprio ad alcuni aspetti biografici la genesi di un'interpretazione che apre spazi ermeneutici importanti e versatili per differenti campi di studio. 